\title{
Comparison of Neurovascular Structures at Risk during Ankle Arthroscopy: A Cadaveric Study
}

\author{
Ramez Sakkab, DPM*, Varsha Salunkhe, DPM, Monica Jung, DPM, MS, Kristina Corley, \\ DPM, Jae Yoon Kim, DPM and Gautam Sowda, BS \\ Completed as Podiatric Medical Student, California School of Podiatric Medicine at Samuel Merritt University, \\ Oakland, CA, USA
}

*Corresponding author: Ramez Sakkab, DPM, 4077 5th Ave, GME Dept - MER 35, San Diego, California, 92103, USA

\begin{abstract}
Arthroscopy has become increasingly common for diagnosis and treatment of ankle joint pathology. The four most common portals used for ankle arthroscopy are the anteromedial (AM), anterolateral ( $A L)$, posteromedial $(P M)$, and posterolateral (PL). And anatomy of neurovascular structures (NVS) along the ankle can significantly vary. The distance of NVS was compared to anatomic landmarks of ankle arthroscopic portals to verify safe zones for scope insertion. Twenty-six fresh-frozen cadavers were utilized, with dissection of standard anatomic landmarks and NVS. Portals were made and verified with a $2.7 \mathrm{~mm}$ arthroscope. Results found significant differences in the comparison of mean distance between anatomic landmarks, with the exception of peroneus tertius to the intermediate dorsal cutaneous nerve (IDCN) $(p=0.181$, all others $p<$ $0.0001)$. By quantifying a scope space, the antero-medial and anterolateral portals had the largest margin of error at 0.82 and $1.04 \mathrm{~cm}$, respectively. The saphenous nerve and vein were an average 1.39 and $1.23 \mathrm{~cm}$ from the anteromedial portal, respectively. Peroneus tertius tendon was an average $0.23 \mathrm{~cm}$ from the IDCN. The tibialis anterior tendon was an average $1.10 \mathrm{~cm}$ lateral from the medial gutter, the peroneus tertius tendon $1.31 \mathrm{~cm}$ medial to the lateral gutter, and Achilles tendon $0.94 \mathrm{~cm}$ and $0.73 \mathrm{~cm}$ from the medial and lateral gutter, respectively. The anterolateral portal had the greatest anatomic variability. This data supports starting with the medial portal to facilitate visualization of lateralsided anatomy prior to anterolateral portal placement.
\end{abstract}

\section{Keywords}

Ankle arthroscopy, Portals, Anatomy, Safe zones

\author{
Abbreviations \\ IDCN: Intermediate Dorsal Cutaneous Nerve; AM: \\ Anteromedial; AL: Anterolateral; PM: Posteromedial; PL: \\ Posterolateral, NVS: Neurovascular Structures; SS: Scope \\ Space
}

\section{Introduction}

Arthroscopy has become increasingly common for diagnosis and treatment of ankle joint pathology [1-3]. The four most common portals used for ankle arthroscopy are the antero-medial (AM), anterolateral $(A L)$, posteromedial $(P M)$, and postero-lateral $(P L)$. All of these run close to neurovascular structures (NVS), making them susceptible to injury during insertion $[3,4]$. A recent meta-analysis found overall complications using anterior or posterior portals to be between 3.4-9\% [5]. The most common being nerverelated injury. The foot and ankle specialist should be familiar with all relevant structures circumferentially across the ankle joint, as well as their relative distance to one another. Allowing for greater reproducibility when placing portals for ankle arthroscopy.

Previous studies have evaluated the distance from portal entry to neurovascular structures, but did not specify the amount of distance available between landmarks for portal entry. And to the author's knowledge, previous reports have not noted the proximity of the ankle gutters. The purpose of this study was to compare the distance of NVS from the four ankle

\footnotetext{
Citation: Sakkab R, Salunkhe V, Jung M, Corley K, Kim JY, et al. (2021) Comparison of Neurovascular Structures at Risk during Ankle Arthroscopy: A Cadaveric Study. Int J Foot Ankle 5:061. doi. org/10.23937/2643-3885/1710061

Accepted: August 28, 2021; Published: August 30, 2021

Copyright: (c) 2021 Sakkab R, et al. This is an open-access article distributed under the terms of the Creative Commons Attribution License, which permits unrestricted use, distribution, and reproduction in any medium, provided the original author and source are credited.
} 


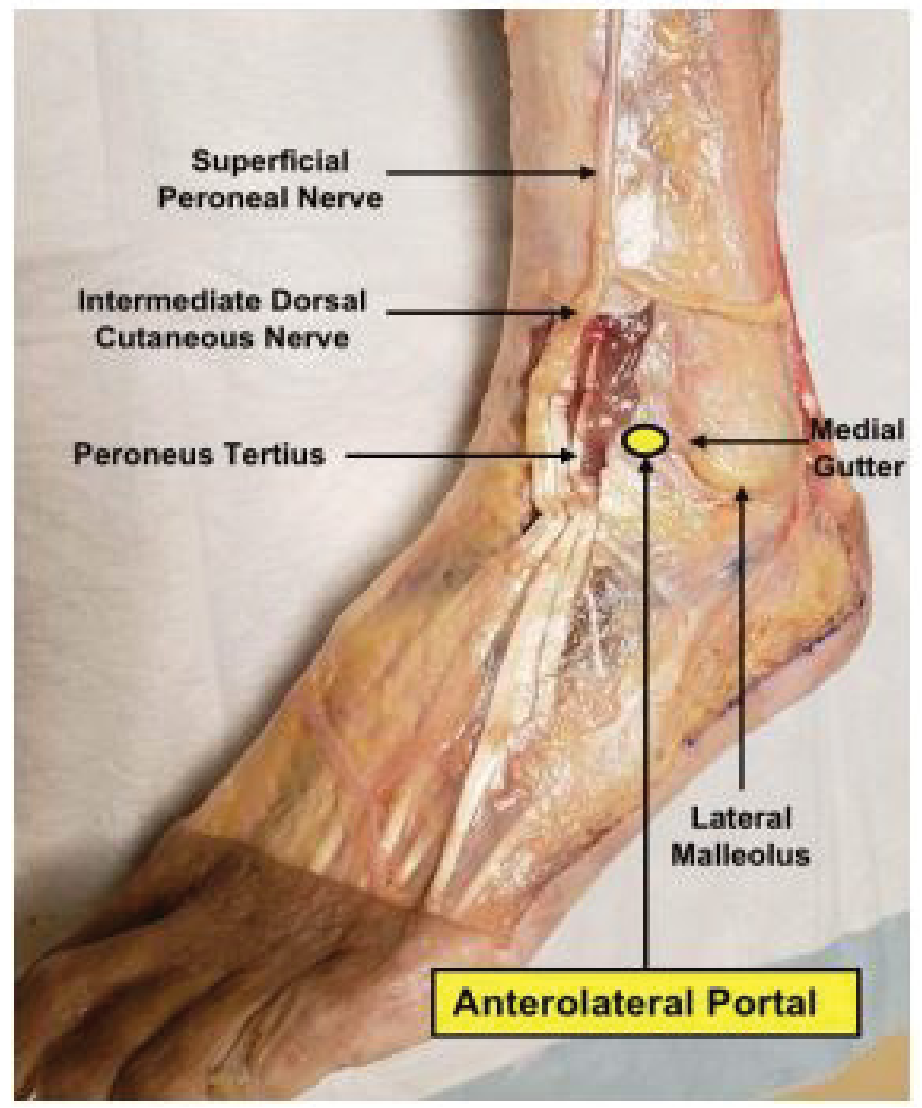

Figure 1: Anterolateral portal located medial to lateral malleolus and lateral to peroneus tertius along with intermediate dorsal cutaneous nerve.

arthroscopic portals landmarks to verify safe zones for scope insertion.

\section{Materials and Methods}

Twenty-six fresh-frozen cadaveric lower extremities were dissected at the four ankle arthroscopic portals. Institutional Review Board approval was waived given the use of cadaveric specimens. Meticulous dissections were performed at each portal site to expose neurovascular structures, while not disturbing them from their original anatomic location. The AM portal was created medial to the tibial is anterior tendon into the medial gutter. The AL portal was created lateral to the peroneus tertius tendon into the lateral gutter (Figure 1). The PM was created medial to the Achilles tendon, and the PL was created lateral to the Achilles tendon into the medial and lateral gutters, respectively. The distances from each anatomical landmark to the neurovascular structures at the level of the ankle joint were measured and recorded. Furthermore, an ankle scope $(2.7 \mathrm{~mm})$ was later placed to ensure proper portal size and precision. All dissections were performed by the same two researchers while all measurements were completed and reviewed by two other researchers to maintain blinding and consistency.

Statistics began with Kruskal-Wallis test to determine parametric and non-parametric data. Descriptive data and comparison of means was conducted with
Wilcoxon signed-rank test and ANOVA as appropriate. All statistical analysis was conducted via SPSS 24.0 (IBM, Armonk, NY).

\section{Results}

All locations demonstrated high variability in juxtaposition to neurovascular structures (Figure 2). The greatest inconsistency was noted between the peroneus tertius (AL portal) and IMDN (mean $22.9 \mathrm{~mm}$ medial, standard deviation of $81.4 \mathrm{~mm}$, range of $30.0 \mathrm{~mm}$ ). The scattered distribution of the anatomy surrounding the AL portal is visualized in Figure 3. Of note, only the distribution of the tibial is anterior to saphenous nerve was normally distributed per Komolgorov-Smirnov testing. However, the differences in variance between landmarks to neurovascular structures or gutters was not significant $(p=0.88)$.

One-sample ANOVA test demonstrated significant differences in the comparison of mean distance between anatomic landmarks, with the exception of peroneus tertius to the IDCN ( $p=0.181$, all others $p<0.0001)$. The values for which are featured in Table 1 . While the range for the IDCN to peroneus tertius was $3.0 \mathrm{~cm}$, no other grouping had a range greater than $2.1 \mathrm{~cm}$.

Moreover, to the authors' knowledge, previous studies have not quantified the distance between landmarks and the ankle joint space. In the present study, the interval between anatomic landmarks and the 


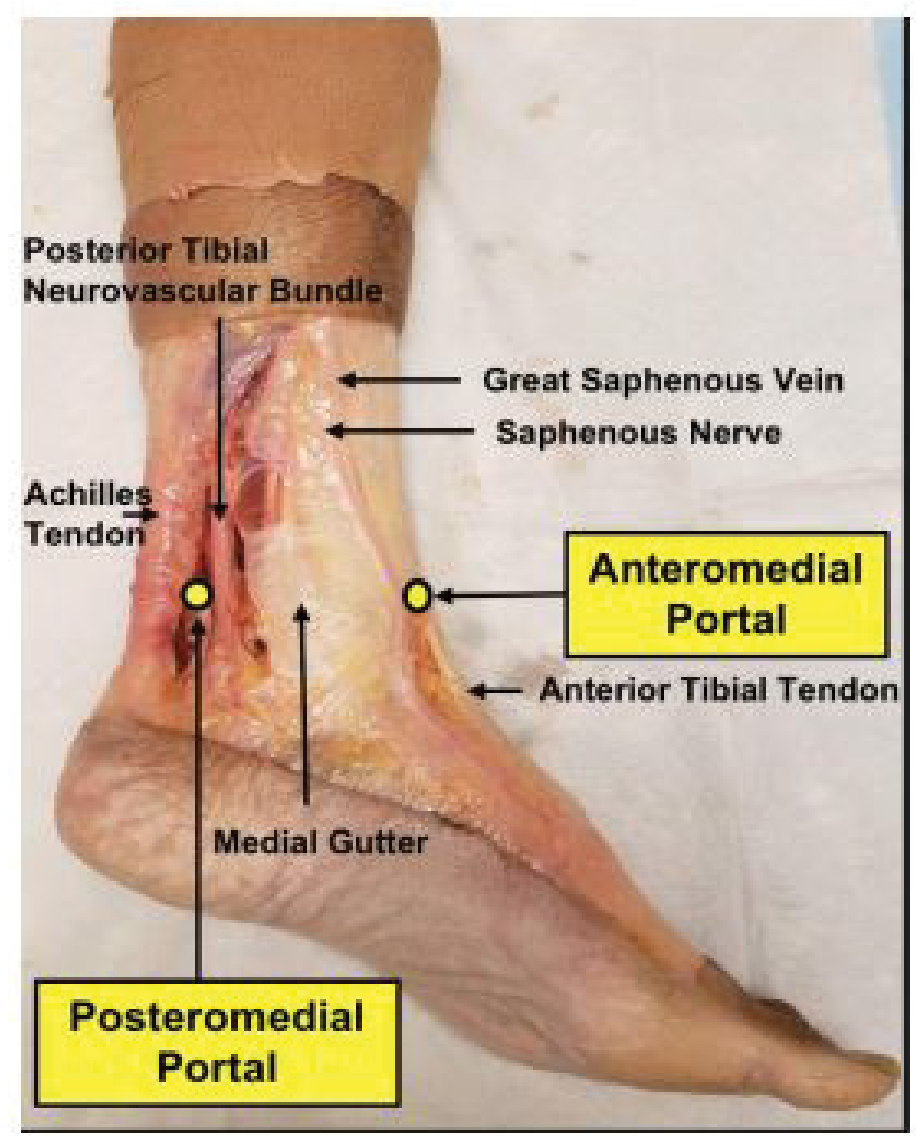

Figure 2: Anteromedial and Posteromedial portals. The AM portal is located medially to tibialis anterior and lateral to the great saphenous vein and saphenous nerve. PM portal is located medial to the AT and lateral to the neurovascular bundle.

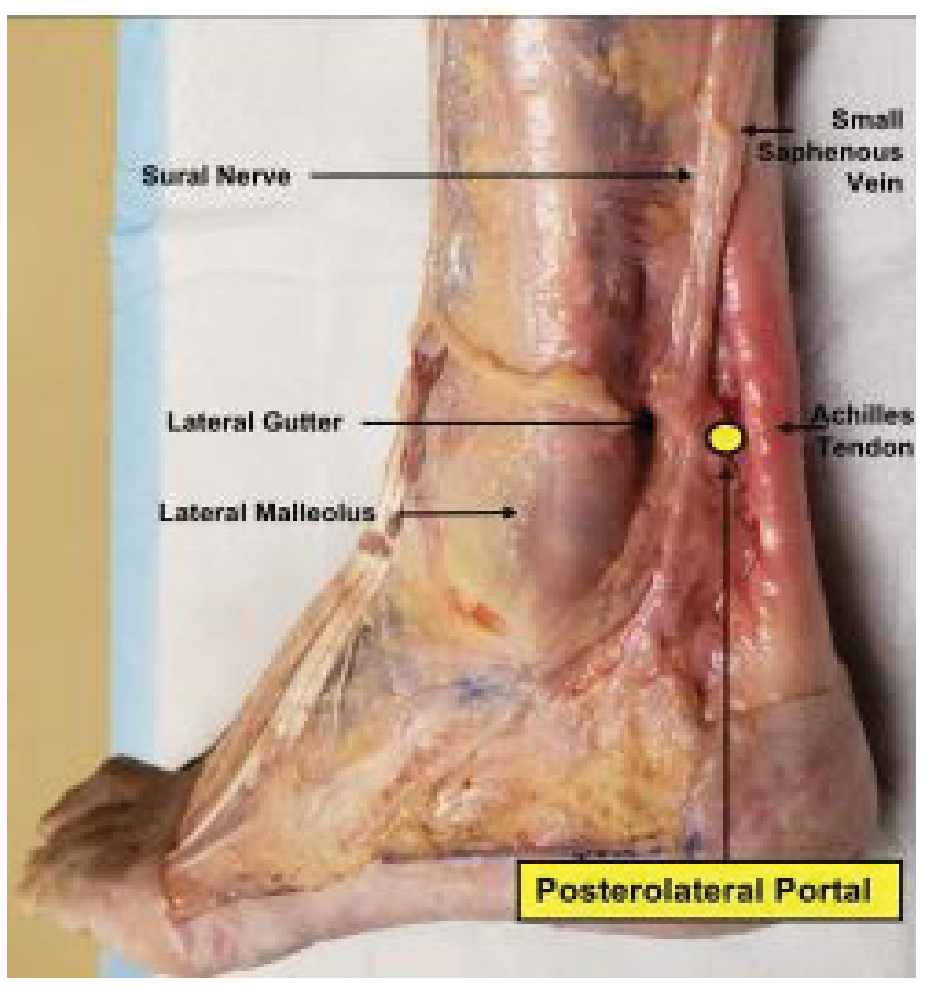

Figure 3: Posterolateral portal dissection located lateral to the AT and medial to sural nerve and small saphenous vein. 
Table 1: Measurements between anatomic landmarks to neural structure or ankle joint gutter.

\begin{tabular}{|l|l|l|}
\hline Portal: Landmark to Structure & Mean (range) (cm) & SE (cm) \\
\hline Anteromedial: & & 0.492 \\
\hline Tibialis Anterior - medial gutter & $1.10(0.5-2.1)$ & 0.412 \\
\hline Tibialis Anterior - saphenous nerve & $1.39(0.5-2.1)$ & 0.539 \\
\hline Tibialis Anterior - saphenous vein & $1.23(0.2-2.2)$ & 0.423 \\
\hline Anterolateral: & & 0.8137 \\
\hline Peroneus Tertius - lateral gutter & $1.31(0.5-2.1)$ & \\
\hline Peroneus Tertius - IDCN & $0.23(-1.2-+1.8)$ & 0.431 \\
\hline Posterolatera: & & 0.411 \\
\hline Tibialis Anterior - lateral gutter & $0.94(0.1-1.8)$ & 0.447 \\
\hline Tibialis Anterior - sural nerve & $0.89(0.1-2.2)$ & 0.449 \\
\hline Posteromedial: & & $0.73(0.2-1.6)$ \\
\hline Achilles Tendon - medial gutter & $0.90(0.1-1.9)$ & \\
\hline Achilles Tendon - tibial nerve & & \\
\hline
\end{tabular}

*Intermediate dorsal cutaneous nerve, mean distance not significantly different against all others $(p=0.181)$; SE: Standard error

ankle gutter was measured. The tibial is anterior tendon was $1.10 \mathrm{~cm}$ lateral from the medial gutter, the peroneus tertius tendon was $1.31 \mathrm{~cm}$ medial to the lateral gutter, the Achilles tendon was $0.94 \mathrm{~cm}$ and $0.73 \mathrm{~cm}$ from the medial and lateral gutter, respectively. Subtracting these lengths by the scope diameter (4.0 or $2.7 \mathrm{~mm}$ ), a resultant scope space (SS) can be quantified. The antero-medial and antero-lateral portals had the largest SS for a $2.7 \mathrm{~mm}$ scope at 0.82 and $1.04 \mathrm{~cm}$ respectively. However, as comparisons of mean SS between the peroneus tertius to IDCN was not significant, the medial portal had the longest SS of statistical significance ( $p$ $<0.0001)$. As such, this portal site has a theoretical greatest amount of available space, and thus the most room for error.

The saphenous and sural nerves were nearly equidistant from the Achilles tendon, at $0.896 \mathrm{~cm}$ medially and $0.8923 \mathrm{~cm}$ laterally, respectively. Additionally, both posterior portals localized a smaller SS than their anterior counterparts. At $0.67 \mathrm{~cm}$ and $0.46 \mathrm{~cm}$ of SS for the posteromedial and posterol ateral portals, respectively.

\section{Discussion}

Ankle arthroscopy is an important, minimally invasive tool for the diagnosis and treatment of numerous pathologies $[1,3,4]$. In comparison with arthroscopic surgery of other joints, neurovascular complications occur more often in association with ankle arthroscopy, emphasizing the importance of determining anatomical safe zones in relation to the 4 portals [2].

The most common complication of ankle arthroscopy is neurologic injury, accounting for up to half of the $9 \%$ complication rate in a study done by Ferkel, et al. [3] Similarly, Deng, et al. encountered an overall incidence of complications of $7.69 \%$, with injury to the superficial peroneal nerve the most prevalent [4]. A meta-analysis of ten cadaveric studies analyzing the neurovascular and tendinous structures at risk in ankle arthroscopy by Yammine, et al. reported a total of 14 nerve injuries; ten of those injuries were to the SPN, with 6 of the injuries incurred through the AL portal [5]. With this information, we hypothesized that the AL portal holds the highest risk for iatrogenic injury to the IDCN.

The anatomic variation of the SPN and its branches are established in the literature [6] and the distance from the SPN to the AL portal is highly varied. Buckingham, et al. measured a distance of $0.5 \pm 2.5 \mathrm{~mm}$, Scheibling, et al. measured a distance of $5.5 \pm 3.5 \mathrm{~mm}$, and Woo, et al. measured a distance of $1.8 \pm 1.25 \mathrm{~mm}$ between the AL portaland the SPN in a collective total of 35 cadavers [6-9]. We hypothesized that the distance from the AL portal to the IDCN would show considerable variation in its anatomic distribution.

The measurements quantified comfortably fall within one standard deviation of those published in a meta-analysis of ankle arthroscopy cadaveric studies per Yammine, et al. [10]. Similar to their work, our results found the intermediate dorsal cutaneous nerve and tendinous structures to be at highest risk of iatrogenic injury. The saphenous nerve's relation to the tibialis anterior tendon (mean $13.9 \mathrm{~mm}$ ) followed a bimodal distribution. Such consistency may corroborate standard technique of first creating the AM portal (Figure 4). In contrast, the IMDN's labile location supports the fact that SPN injury is one of the most frequent complications of ankle $[4,11]$. Furthermore, the results reinforce the technique of antero-medial portal creation first, followed by better visualization of lateral anatomy. Five specimen's IDCN were located lateral to the portal, rather than the archetypal medial distribution. Undoubtedly, these variants contributed significantly to the mean distance of $2.3 \mathrm{~mm}$ of the AL portal to the IDCN. Other anatomic variants may 


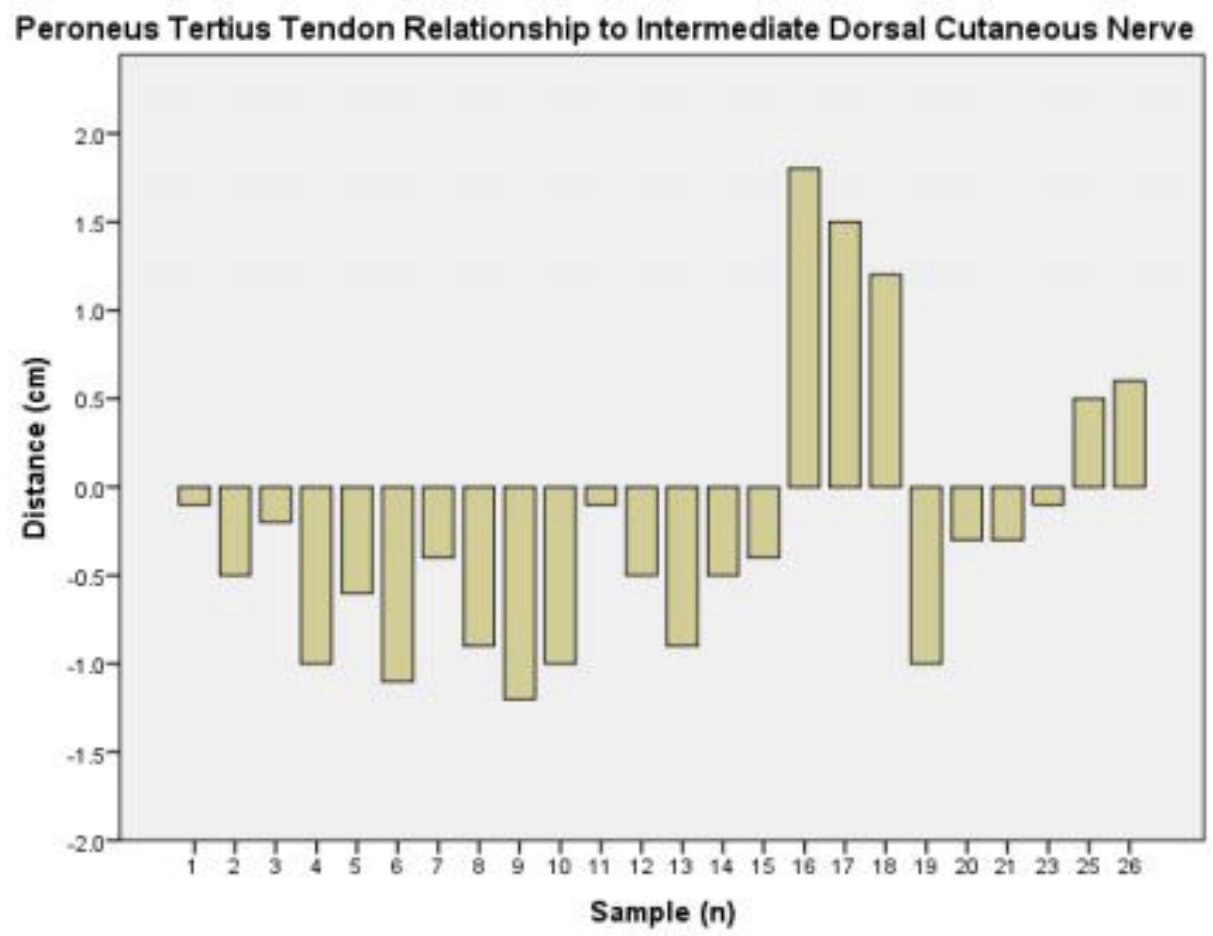

Figure 4: Histogram illustrating high variability of intermediate dorsal cutaneous nerve in relation to peroneus tertius near the anterolateral portal. Positive values represent lateral distribution, negative medial.

have existed. One nascent concern is having falsely identified a medial dorsal cutaneous or undivided superficial peroneal nerve as the IDCN. However, no variant branches of the perforating peroneal or anterior tibial artery were identified, as have been described previously [12]. Overall, the considerable number of cadaveric specimens $(n=26)$ contributed to the statistical significance achieved in comparative analysis. This number is greater than that founds in two recent reports that contained varied results $[13,14]$.

The present study had clear limitations, including but not limited to: methodologic criteria, potential errors in consistency of measurements, and modifications to cadaveric limbs. Increased accuracy may have been obtained by averaging the measurements of multiple observers. Of note, five of $26(19 \%)$ cadaveric limbs had fiber wire and bone anchors within the posterosuperior calcaneus. Neurovascular structures of these limbs were evaluated to ensure they were undisturbed prior to inclusion. Other limitations are those inherent to investigations pertaining to fresh-frozen cadavers, a constrained sample size, and sample heterogeneity.

In conclusion, our results suggest that landmarks near the antero-medial portal demonstrate the least anatomic variance, whereas those near to the anterolateral demonstrate the greatest. The four most common portals of ankle arthroscopy have a mean of 7.3 $\mathrm{mm}$ to $13.1 \mathrm{~mm}$ of length between anatomic landmark and adjacent ankle joint gutter. The present data can be utilized by foot and ankle surgeons when planning portal placement and analyzing the complications thereof.

\section{Acknowledgements}

We would like to formally thank Matthew Smith and Arthrex for providing the cadaveric specimens. We sincerely appreciate the time, guidance and support from Dr. Barbara Guzman, Dr. Mary Premenko-Lanier, and Dr. Reed Rowan for this project. All authors report they have no conflicts of interest related to this work.

\section{References}

1. Ferkel RD, Chams RN (2007) Chronic lateral instability: Arthroscopic findings and long-term results. Foot Ankle Int 28: 24-31.

2. Cugat R, Ares O, Cuscó X, Garcia M, Samitier G, et al. (2008) Posterior tibial nerve lesions in ankle arthroscopy. Arch Orthop Trauma Surg 128: 485-487.

3. Ferkel RD, Small HN, Gittins JE (2001) Complications in foot and ankle arthroscopy. Clin Orthop Relat Res 391: 89104.

4. Deng DF, Hamilton GA, Lee M, Rush S, Ford LA, et al. (2012) Complications associated with foot and ankle arthroscopy. J Foot Ankle Surg 51: 281-284.

5. Zekry M, Shahban SA, El Gamal T, Platt S (2019) A literature review of the complications following anterior and posterior ankle arthroscopy. Foot Ankle Surg 25: 553-558.

6. Ucerler H, Ikiz ZAA, Uygur M (2007) A Cadaver study on preserving peroneal nerves during ankle arthroscopy. Foot Ankle Int 28: 1172-1178.

7. Buckingham RA, Winson IG, Kelly AJ (1997) An anatomical study of a new portal for ankle arthroscopy. J Bone Joint Surg 79: 650-652.

8. Scheibling B, Koch G, Clavert P (2017) Cadaver study of anatomic landmark identification for placing ankle arthroscopy portals. Orthop Traumatol Surg Res 103: 387-391. 
9. Woo SB, Wong TM, Chan WL, Yen CH, Wong WC, et al. (2010) Anatomic variations of neurovascular structures of the ankle in relation to arthroscopic portals: A cadaveric study of chinese subjects. J Orthop Surg 18: 71-75.

10. Yammine K, Assi C (2018) Neurovascular and tendon injuries due to ankle arthroscopy portals: A meta-analysis of interventional cadaveric studies. Surg Radiol Anat 40: 489-497.

11. Oliva XM, López JMM, Planella MM, Bravo A, Rodrigues Pinto R (2015) Anatomical relations of anterior and posterior ankle arthroscopy portals: A cadaveric study. Eur J Orthop Surg Traumatol 25: 577-581.
12. Jeon A, Seo CM, Lee JH, Han SH (2018) The distribution pattern of the neurovascular structures for anterior ankle arthroscopy to minimize structural injury: Anatomical Study. Bio Med Res Int 2018: 1-5.

13. Gohiya A, Karoria A, Alawa S, Khare A (2020) Safety of neurovascular structures in ankle arthroscopy: Cadaveric study. Int J Orthop Sci 6: 625-627.

14. Pitts CC, McKissack HM, Anderson MC, Buddemeyer KM, Bassetty C, et al. (2020) Anatomical structures at risk in the arthroscopic Broström-Gould procedure: A cadaver study. Foot Ankle Surg 26: 343-346. 